\title{
Intra- and Inter-Observer Reproducibility of Ultrasound Measurements of Pelvic Inlet Diameter in Pregnant Women
}

\author{
Yi Man Wah, Yiu Man Chan, Daljit Singh Sahota, Shuk Yi Hui, Tze Kin Lau, \\ Tak Yeung Leung \\ Department of Obstetrics and Gynecology, Prince of Wales Hospital, The Chinese University of Hong Kong, \\ Hong Kong, China \\ Email: isabellawah@cuhk.edu.hk
}

Received 19 June 2014; revised 15 July 2014; accepted 10 August 2014

Copyright (C) 2014 by authors and Scientific Research Publishing Inc. This work is licensed under the Creative Commons Attribution International License (CC BY). http://creativecommons.org/licenses/by/4.0/

\section{(c) (i) Open Access}

\begin{abstract}
Objectives: To determine intra- and inter-observer reproducibility of the ultrasound measurement of pelvic inlet in the first trimester of pregnancy. Methods: Transabdominal pelvic ultrasound was performed to measure the pelvic inlet anteroposterior diameter using a $2 \mathrm{D}$ probe in twelve Chinese women having a singleton pregnancy. Scans were performed in the first trimester by three sonographers. The pelvic inlet anteroposterior diameter was defined as the distance between the anterior surfaces of the sacral promontory and the superior medial border of the pubic bone most adjacent to the pubic symphysis. Intra-observer repeatability was determined and analysis of variance was performed to assess inter-observer measurements. The bias between any two sonographers' measurements was assessed by calculating the $95 \%$ confidence interval for the mean difference between sonographers paired measurements. Results: Intra-observer reproducibility was $0.71 \mathrm{~cm}$. Analysis of variance indicated that there was no significant difference between sonographers' measurements $(p=0.46)$. The bias between two sonographers' measurements ranged from 0.05 to $0.32 \mathrm{~cm}$. Conclusion: It is technically feasible to measure the pelvic inlet diameter using ultrasound (USG) at the first trimester. High inter-observer reproducibility can be achieved. Further studies are required to establish the potential role of this technique and the measurement of the pelvic inlet diameter in prediction of labor outcome.
\end{abstract}

\section{Keywords}

Pelvimetry, Pelvic Inlet Diameter, Obstetric Conjugate 


\section{Introduction}

Cephalopelvic disproportion or contracted maternal pelvis is one of the major causes of arrest of progress or obstructed labor. It is associated with significant maternal and perinatal morbidities [1]. Several modalities have been proposed to measure the maternal pelvic size antenatally in order to predict labor outcome. None however, has yet been found to be useful in routine practice. Clinical pelvimetry, estimation of pelvic adequacy by digital examination, can be performed easily at the bedside. However, physical examination is subjective, and is difficult to be standardized; digital examination only allows assessment of the diagonal conjugate of the inlet instead of the true inlet. Other assessment modalities include X-ray pelvimetry and Magnetic Resonance Imaging (MRI) pelvimetry. Whilst X-ray pelvimetry allows for better determination of pelvic dimension, a Cochrane systematic review concludes that there is insufficient evidence to support its routine use in women with fetuses in cephalic presentation [2]. MRI produces a high-quality image allowing accurate measurement of the bony pelvis. One prospective study has demonstrated how MRI could be used to identify women at risk for caesarean section by identifying women with abnormalities on pelvimetry [3]. MRI has also been used to provide improved selection of the route of delivery at term for fetuses in breech presentation, resulting in significant reduction in the emergency caesarean section rate in the study group compared to the controls without compromising neonatal outcome [4]. Nevertheless, the cost of MRI, routine availability and patient's willingness to undergo a scan limit its application in routine clinical practice.

Ultrasound has potential advantages over MRI and X-rays as it is readily available at the bedside, allows viewing at different angles and does not carry the risk of radiation exposure to the fetus and the mother. Its role in assessing the maternal pelvis however has been very limited mainly because of the poor imaging quality. With the recent advances in ultrasound technology, the differentiation between different tissues is much better and the limitation may be overcome.

In this study we developed a technique to measure the pelvic inlet, and assessed the intra-observer repeatability and inter-observer reproducibility with measurements using this technique.

\section{Methods}

Women with singleton pregnancy in the first trimester (11 - 13 weeks and 6 days of gestation) were invited to undergo a transabdominal ultrasound examination of the pelvic inlet between November 2010 and December 2010. Women who consented to participate were examined in a supine position using a 2D probe with a sector angle of 107 degrees and a GE V8 ultrasound machine. The scans were performed by 3 sonographers (A to C) who all had 3 or more years of experience performing obstetric ultrasound scan. At the onset of the examination the ultrasound probe was placed in the sagittal position just below the umbilicus, and then gradually moved downwards to the pubic symphysis. The sacral promontory was identified as the point where the lumbar vertebra and the sacrum meet on the sagittal plane. The anterior surface of the sacral promontory was fixed as the posterior landmark which appears in the left lower part of the image. The superior medial border of the pubic bone where most adjacent to the pubic symphysis on either left or right side was used as the anterior landmark instead of the inter-pubic disc as it was not clearly identifiable on ultrasound. The distance between these two landmarks was regarded as the pelvic inlet anteroposterior diameter (Figure 1). Measurements were performed by the three sonographers for all patients. Sonographers were instructed to capture a new image on each measurement occasion. Sonographer A was instructed to perform three consecutive scans and measurements on each patient for assessment of within sonographer repeatability. All measurements were taken at a single examination for the same patient with no break. The measurement results were blinded to the sonographer during the examination by placing a non-opaque cutout template on the screen over the region where measurements were displayed.

Intra-observer repeatability was assessed by calculating the repeatability coefficient [5]. The repeatability coefficient quantifies how much the absolute difference between measurements on a subject would be expected to differ by on $95 \%$ of occasions. A one way analysis of variance (ANOVA) was firstly performed on the repeated measurements to estimate the within subject standard deviation (SDWS). The repeatability coefficient was determined using the formula $\sqrt{2} \times 1.96 \times$ SDWS.

Analysis of variance was performed to determine if a significant difference existed between inter-observer measurements. The mean of the paired difference (bias) and 95\% confidence interval (CI) were determined and Bland Altman plots were created for each pair of sonographers [6]. The intraclass correlation (ICC) was used to measure the reliability of the sonographers' measurements. 


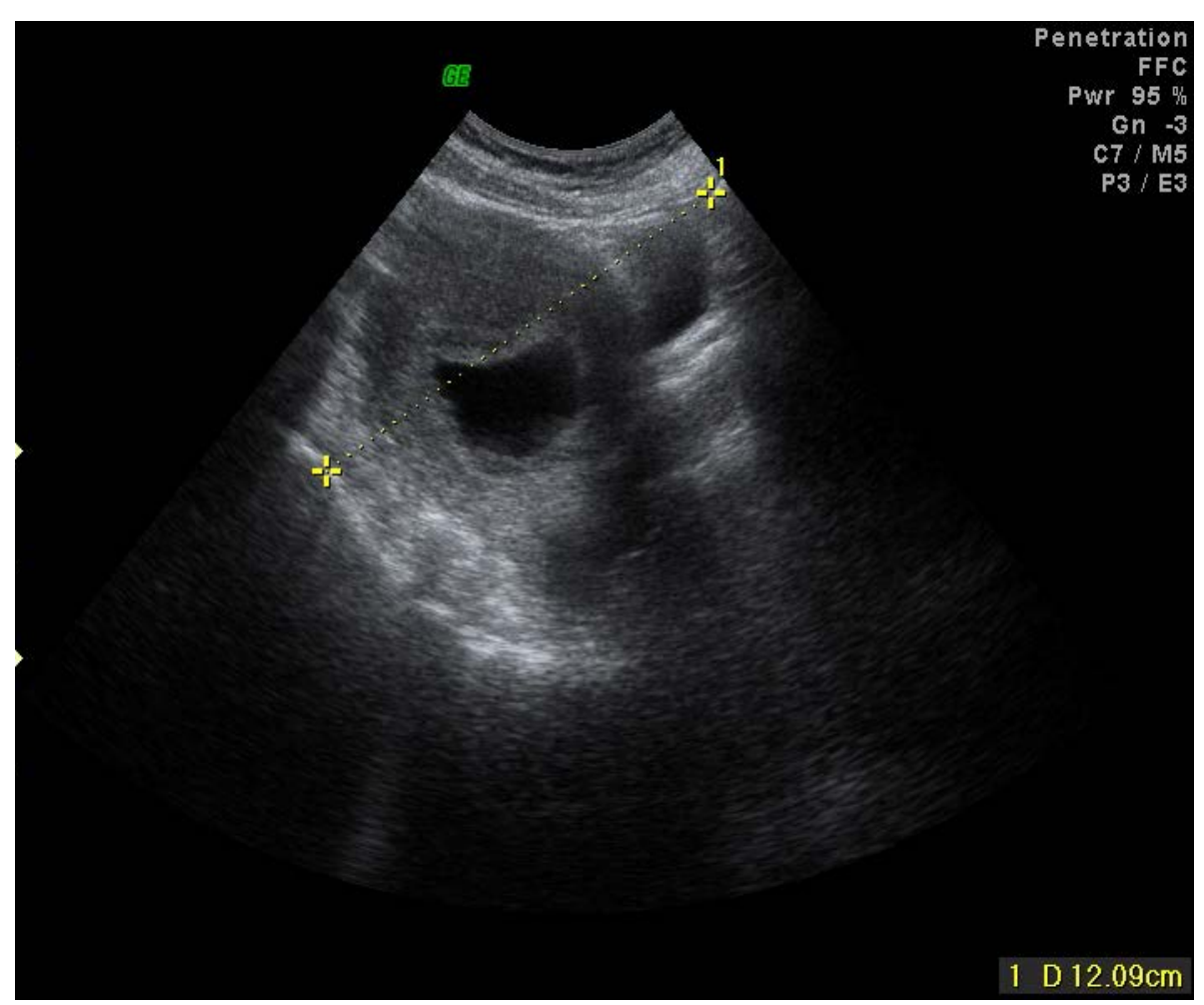

Figure 1. Ultrasound image of the pelvis showing the pelvic inlet diameter measurement.

The statistical package for social sciences for Windows version 17.0 (SPSS, Inc, Illinois, USA) and Excel 2003 (Microsoft Corporation, USA) were used to perform statistical and graphical analysis of all data. A “p” value of 0.05 or lower was considered statistically significant. The study was approved by the Institutional Ethics Review Board.

\section{Results}

Twelve Chinese women having a singleton pregnancy agreed to participate in the study. The median maternal age at estimate date of confinement was 32.5 years (Interquartile range (IQR): 20 to 38 years) and 5 (41.7\%) were nulliparous. The number of women assessed who were in their 11th and 12th week of gestation was 3 and 9 respectively.

Pelvic inlet measurements were successfully recorded in all 12 women using the reported measurement protocol. Figure 1 shows a typical example of the type of image obtained by sonographers when measuring the pelvic inlet diameter. The pelvic inlet diameter measurements ranged from 9.9 to $14.96 \mathrm{~cm}$. Table 1 presents the minimum, maximum, mean and standard deviation (SD) values for the pelvic inlet diameter for each of the three sonographers performing the scans.

Intra-observer reproducibility for sonographer A was $0.71 \mathrm{~cm}$. Table 2 and Figures 2(a)-2(c) present the comparison of the inter-observer agreement between the pairs of sonographer measurements. ANOVA showed that there is no significant difference in measurement between sonographers $(p=0.46)$ and the ICC was 0.76 . The bias between any two pairs of sonographers ranged from 0.05 to $0.33 \mathrm{~cm}$.

\section{Discussion}

Prediction and identification of women who are able to achieve a successful vaginal delivery remains a question for obstetricians. One reason for failure to have a vaginal delivery is an inadequate pelvis. Unrecognised cephalopelvic disproportion can lead to a failed instrumental delivery and or second stage caesarean section which imposes high morbidity to both the fetus and the mother. Keller et al. showed that the obstetric conjugate was lower in women with cephalopelvic disproportion compared to those who had a vaginal delivery [7]. Mari et al. 
Table 1. Descriptive statistics for pelvic inlet diameter for each of the three sonographers.

\begin{tabular}{ccccc}
\hline & \multicolumn{3}{c}{ Pelvic inlet diameter $(\mathrm{cm})$} \\
\hline Sonographer & Minimum & Maximum & Mean & SD \\
\hline A & 11.31 & 14.50 & 12.49 & 0.75 \\
B & 10.14 & 14.96 & 12.38 & 1.29 \\
C & 9.90 & 14.27 & 12.23 & 0.89 \\
\hline
\end{tabular}

Table 2. Descriptive statistics for all possible pairs of sonographer pelvic inlet diameter measurements.

\begin{tabular}{cccccc}
\hline & \multicolumn{5}{c}{ Pelvic inlet diameter differences (cm) } \\
\cline { 2 - 6 } Sonographer pairs & Minimum & Maximum & Mean & $(95 \%$ CI $)$ & SD \\
\hline A and B & -2.01 & 2.02 & 0.05 & $(-2.09$ to 2.19$)$ & 1.09 \\
A and C & -1.57 & 2.45 & 0.33 & $(-1.86$ to 2.52$)$ & 1.12 \\
B and C & -0.31 & 1.75 & 0.28 & $(-0.87$ to 1.42$)$ & 0.58 \\
\hline
\end{tabular}

also demonstrated that the percentage of patients requiring caesarean section for dystocia was significantly higher in women with an obstetric conjugate of less than $12 \mathrm{~cm}(50 \%$ vs $7.1 \%, \mathrm{p}<0.001)$ [8]. Although not all pelvic plane diameters can be measured by ultrasound, measurement of the pelvic inlet may provide obstetricians with more information in determining the likelihood that a woman will have a successful vaginal delivery.

Ultrasound is now routinely and widely used in modern obstetric practice. However despite its wide spread availability, the role of ultrasound to assess the pelvic inlet size has not been evaluated. Our analysis indicates that experienced sonographers could achieve good intra-observer repeatability when measuring the pelvic inlet. The inter-observer reproducibility was also high as the ICC was 0.86 (95\% CI $0.73-0.93)$. The reproducibility was similar to the intra-observer and inter-observer reliability of pelvic inlet diameter measurements achieved by MRI pelvimetry in previous studies [7] [9]. Our study demonstrated that the inter-observer agreement is promising and that our proposed method of measuring pelvic inlet of pregnant women in the first trimester is clear and sufficiently simple that sonographers were able to perform with the minimal of instructions. Further studies are however needed to determine 1) whether an intra-observer repeatability of $0.71 \mathrm{~cm}$ is clinically acceptable and 2) whether routine ultrasound pelvimetry will reliably identify women with an inadequately sized pelvic inlet.

There are some limitations with our study. Firstly, we did not perform imaging of the pubic symphysis directly as the pubic symphysis is difficult to visualize using ultrasound. We used the superior medial border of the pubic bone as the landmark instead of the pubic symphysis for the measurement. As a result the anteroposterior diameter might be slightly longer than the measurements obtained using X-ray, CT or MRI as the measurement is not a straight line from the sacral promontory to the pubic symphysis. Mari et al. also demonstrated that the measurement of pelvic inlet using ultrasound was possible. Their study method was similar to our study except that the pubic bone was identified before the identification of the sacral promontory [8]. This study showed that the mean ultrasonic pelvic inlet measurement was longer than the mean radiographic pelvic inlet measurement at 36 weeks of gestation. However, there was a positive correlation between the pelvic inlet measurement using ultrasound and X-ray [8]. Therefore, the use of ultrasound in pelvic inlet measurement should still be useful provide that suitable reference curves are established. Secondly, we did not compare individual sonographers' measurements of the pelvic inlet diameter with other measurement modalities such as MRI. Thus the difference in the pelvic inlet diameter using ultrasound and other imaging modalities cannot be determined. Thirdly, our sample size is small. A larger cohort would be needed to establish the normal reference ranges as well as robust and clinically useful criteria for the identification of women with cephalopelvic disproportion.

Pelvic inlet measurement using ultrasound in the first trimester is easier as the ultrasound beam might be obscured by fetal bony parts or engaged fetal head in later gestation. During the late first trimester, the gravid uterus occupies the whole pelvic cavity and displaces the bowels away. The amniotic fluid provides a very good medium for the transmission of ultrasound waves to the sacrum. The fetal bones are also less ossified in the early 


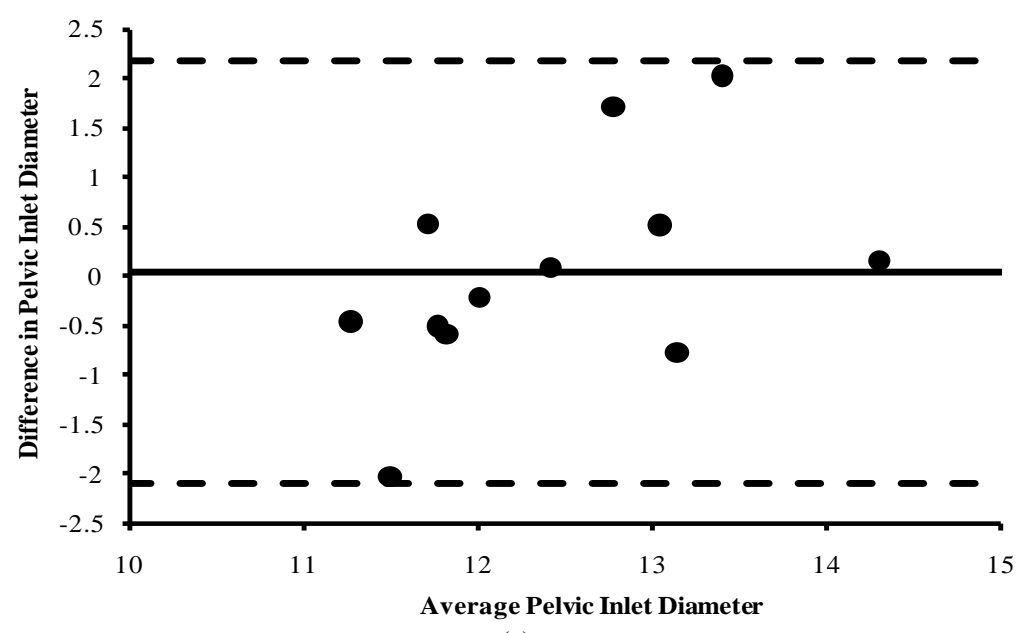

(a)

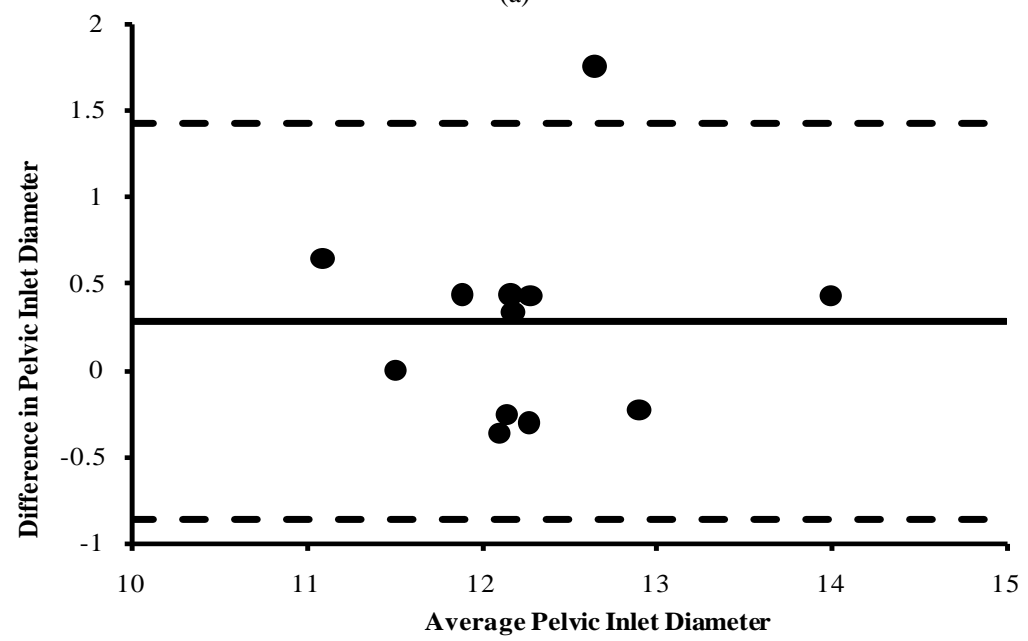

(b)

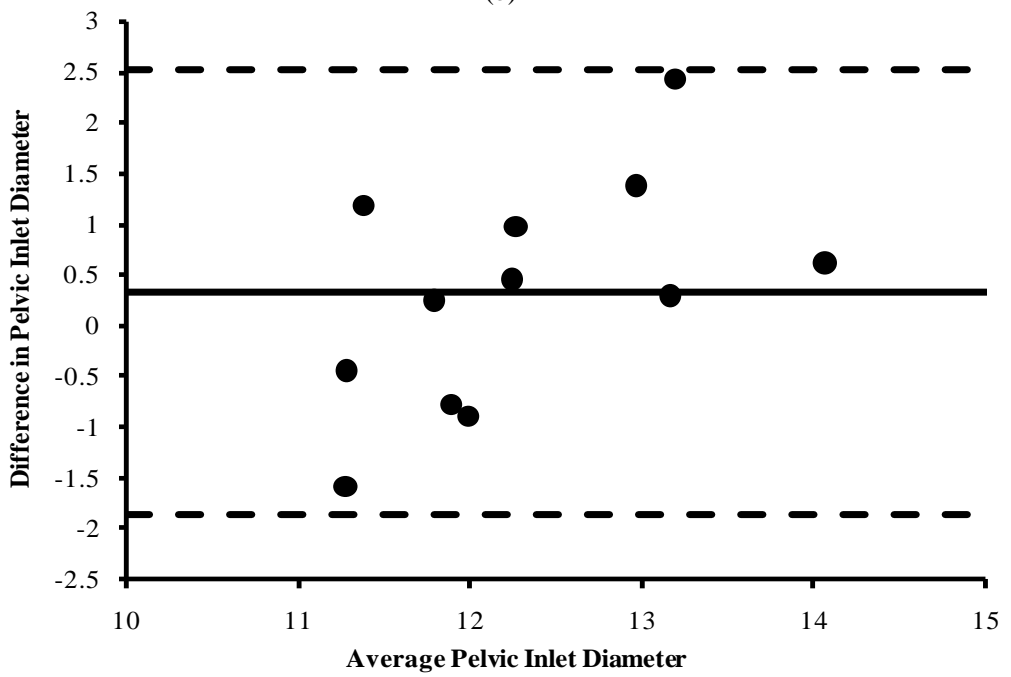

(c)

Figure 2. (a) Sonographer A versus sonographer B. (b) Sonographer B versus sonographer C. (c) Sonographer A versus sonographer C. Pelvic inlet diameter inter-sonographer difference between plotted against the mean of pairs of measurements with the mean difference ( - ) and $95 \%$ limits of agreement (- - - ) indicated. 
gestation so that it is possible to obtain a good image of the sacrum as the landmarks of the pelvic inlet. Although there was no study to evaluate whether the pelvic inlet diameter altered during the antenatal period, a study did show that the pelvic inlet measurements were stable before and after delivery [10]. Thus we can postulate that the pelvic inlet diameter does not change during the antenatal period. The pelvic inlet diameter in our studied Chinese women ranged from 9.9 to $14.96 \mathrm{~cm}$ (mean $12.36 \mathrm{~cm}$ ) which is comparable to the previous study with the measurement of 10.7 to $15.1 \mathrm{~cm}$ (mean $12.9 \mathrm{~cm}$ ) at 28 weeks of pregnancy and 10.7 to $15.1 \mathrm{~cm}$ (mean $12.77 \mathrm{~cm}$ ) at 36 weeks of pregnancy [8]. True conjugate of the pelvic inlet is the most important diameter of the pelvic inlet since it is the shortest distance between the sacrum and the pubic symphysis. It is one of the determining factors of fetal head engagement. Also, the pelvic inlet diameter was found to be significantly smaller for patient with fetal pelvic disproportion compared to those who can achieve spontaneous vaginal delivery [5].

\section{Conclusion}

In conclusion, it is technically feasible to measure the pelvic inlet diameter using USG at the first trimester. High inter-observer reproducibility can be achieved. Further studies are required to establish the potential role of this technique and the measurement of pelvic inlet diameter in prediction of the labor outcome.

\section{References}

[1] Stewart, K.S. and Philpott, R.H. (1980) Fetal Response to Cephalopelvic Disproportion. BJOG: An International Journal of Obstetrics \& Gynaecology, 87, 641-649.

[2] Pattinson, R.C. (2000) Pelvimetry of Fetal Cephalic Presentations at or Near Term. Cochrane Database of Systematic Reviews, 2, Article ID: CD000161.

[3] Sporri, S., Hänggi, W., Braghetti, A., Vock, P. and Schneider, H. (1997) Pelvimetry by Magnetic Resonance Imaging as a Diagnostic Tool to Evaluate Dystosia. Obstetrics Gynecology, 89, 902-908. http://dx.doi.org/10.1016/S0029-7844(97)00148-8

[4] Van Loon, A.J., Mantingh, A., Serlier, E.K., Kroon, G., Mooyaart, E.L. and Huisjes, H.J. (1997) Randomised Controlled Trial of Magnetic-Resonance Pelvimetry in Breech Presentation at Term. Lancet, 350, 1799-1804. http://dx.doi.org/10.1016/S0140-6736(97)05431-7

[5] Bartlett, J.W. and Frost, C. (2008) Reliability, Repeatability and Reproducibility: Analysis of Measurement Errors in Continuous Variables. Ultrasound in Obstetrics Gynecology, 31, 466-475. http://dx.doi.org/10.1002/uog.5256

[6] Bland, J.M. and Altman, D.G. (2003) Applying the Right Statistics: Analyses of Measurement Studies. Ultrasound in Obstetrics Gynecology, 22, 85-93. http://dx.doi.org/10.1002/uog.122

[7] Keller, T.M., Rake, A., Michel, S.C., Seifert, B., Efe, G., Treiber, K., Huch, R., Marincek, B. and Kubik-Huch, R.A. (2003) Obstetric MR Pelvimetry: Reference Values and Evaluation of Inter- and Intraobserver Error and Intraindividual Variability. Radiology, 227, 37-43. http://dx.doi.org/10.1148/radiol.2271011658

[8] Katanozaka, M., Yoshinaga, M., Fuchiwaki, K. and Nagata, Y. (1999) Measurement of Obstetric Conjugate by Ultrasonic Tomography and Its Significance. American Journal of Obstetrics Gynecology, 180, 159-162. http://dx.doi.org/10.1016/S0002-9378(99)70168-7

[9] Korhonen, U., Solja, R., Laitinen, J., Heinonen, S. and Taipale, P. (2010) MR Pelvimetry Measurements, Analysis of Inter- and Intra-Observer Variation. European Journal of Radiology, 75, 56-61. http://dx.doi.org/10.1016/j.ejrad.2009.11.018

[10] Huerta-Enochian, G.S., Katz, V.L., Fox, L.K., Hamlin, J.A. and Kollath, J.P. (2006) Magnetic Resonance-Based Serial Pelvimetry: Do Maternal Pelvic Dimensions Change during Pregnancy? American Journal of Obstetrics Gynecology, 194, 1689-1695. http://dx.doi.org/10.1016/j.ajog.2006.03.008 
Scientific Research Publishing (SCIRP) is one of the largest Open Access journal publishers. It is currently publishing more than 200 open access, online, peer-reviewed journals covering a wide range of academic disciplines. SCIRP serves the worldwide academic communities and contributes to the progress and application of science with its publication.

Other selected journals from SCIRP are listed as below. Submit your manuscript to us via either submit@scirp.org or Online Submission Portal.
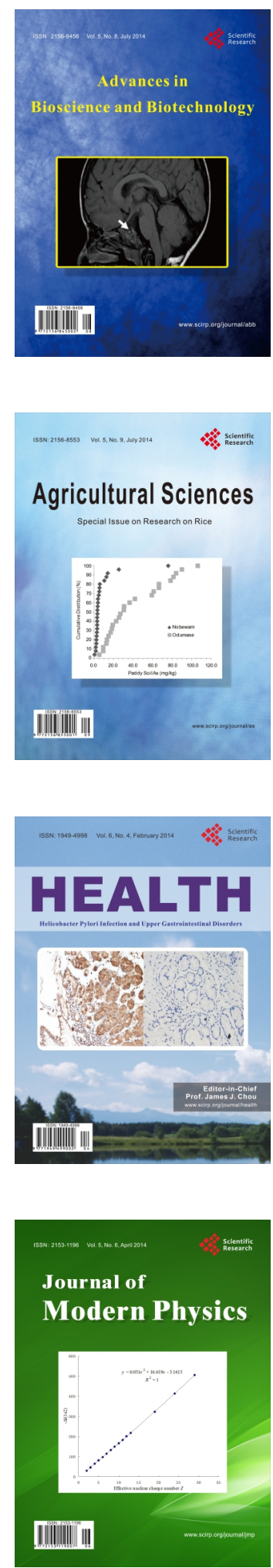
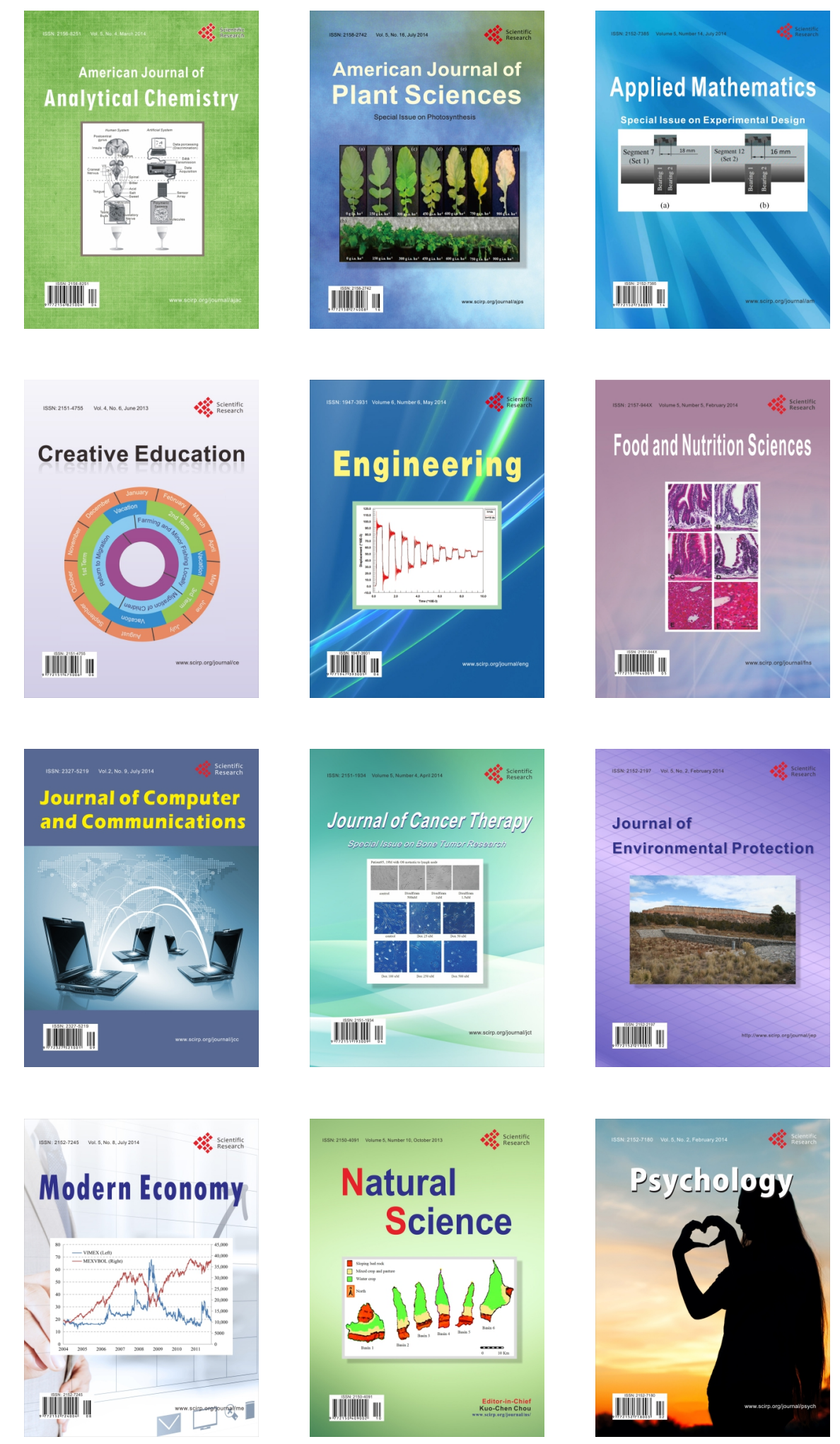